Check for updates

Cite this: RSC Adv., 2019, 9, 6708

\title{
An ensemble variable selection method for vibrational spectroscopic data analysis $\uparrow$
}

\begin{abstract}
Jixiong Zhang, (DD a Hong Yan, ${ }^{a}$ Yanmei Xiong, ${ }^{a}$ Qianqian Li ${ }^{b}$ and Shungeng Min*a
Wavelength selection is a critical factor for pattern recognition of vibrational spectroscopic data. Not only does it alleviate the effect of dimensionality on an algorithm's generalization performance, but it also enhances the understanding and interpretability of multivariate classification models. In this study, a novel partial least squares discriminant analysis (PLSDA)-based wavelength selection algorithm, termed ensemble of bootstrapping space shrinkage (EBSS), has been devised for vibrational spectroscopic data analysis. In the algorithm, a set of subsets are generated from a data set using random sampling. For an individual subset, a feature space is determined by maximizing the expected 10 -fold cross-validation accuracy with a weighted bootstrap sampling strategy. Then an ensemble strategy and a sequential forward selection method are applied to the feature spaces to select characteristic variables. Experimental results obtained from analysis of real vibrational spectroscopic data sets demonstrate that the ensemble wavelength selection algorithm can reserve stable and informative variables for the final modeling and improve predictive ability for multivariate classification models.
\end{abstract}

Received 23rd October 2018

DOI: $10.1039 / \mathrm{c} 8 \mathrm{ra} 08754 \mathrm{~g}$

rsc.li/rsc-advances

data. ${ }^{8,9}$ To data, much effort has been made to improve the performance of PLS-DA, and variable selection has been shown to be one of the most effective ways because there is a close connection between PLS dimension reduction and variable selection. ${ }^{10}$

A number of algorithms for variable selection in the PLS-DA model have been proposed..$^{5,11-22}$ In general, these methods can be classified into three categories and include filter, wrapper and embedded techniques. ${ }^{23}$ Wrapper is the most commonly used technique, because this technique is easy to implement and the interaction between the feature subset search and the classifier is considered. In wrapper methods, a search procedure in the space of the possible feature subset is defined and various subsets of features are generated and evaluated. The evaluation of a specific subset of features is obtained by a PLSDA model. Examples of such methods are backward variable elimination PLS-DA, ${ }^{21}$ moving window PLS-DA ${ }^{22}$ and artificial intelligent algorithm based PLS-DA. ${ }^{6,16,17,19}$ Recently a bootstrapping strategy coupled with model population analysis was used to search for an optimal variable subset in PLS regression models. ${ }^{24}$ In this algorithm, various variable subspaces are generated by the weighted bootstrap sampling (WBS) method. Variables with larger absolute values of PLS regression coefficients are extracted and given a higher sampled weight using model population analysis. Whole variable space shrinks gradually until it becomes an optimal variable subset. A similar approach was also used in a study on spectral interval combination optimization..$^{25}$

In the case of the wrapper variable selection methods guided by a random search, however, a common problem is that they have a high risk of randomicity ${ }^{26}$ given that the probability of finding a suitable model may sometimes happen by chance

${ }^{a}$ College of Science, China Agricultural University, No. 2, Yuanmingyuanxi Road, Haidian District, Beijing 100193, P.R. China. E-mail: minsg@cau.edu.cn; Tel: +86-010-62733091

${ }^{b}$ School of Marine Science, China University of Geosciences in Beijing, Beijing 100086, China

$\dagger$ Electronic supplementary information (ESI) available. See DOI: $10.1039 / \mathrm{c} 8 \mathrm{ra} 08754 \mathrm{~g}$ 
(due to random correlations). Clearly, the stability and reliability of selected results needs to should be strengthened. ${ }^{27}$

In several notable papers that are concerned with ensemble methods in machine learning, ${ }^{28-31}$ a set of classifiers whose individual decisions are combined in some way (typically by weighted or unweighted voting) has been shown to provide improved classification accuracy. Inspired by this, we have made the assumption that an ensemble of variable selection methods may be used to extract the most informative and significant variables and to reduce the stochastic risk. In fact, there have been some reports on the use of ensemble methods for variable selection in chemometrics. For instance, Han et $a .^{32}$ developed a method using an ensemble of Monte Carlo uninformative variable elimination to improve the stability and reliability of selected variables, and Zheng et $a .^{26}$ used an improved version of a voting genetic algorithm (GA) to overcome the stochastic risk of a GA.

In this study, a new PLSDA-based wavelength selection algorithm, termed ensemble of bootstrapping space shrinkage (EBSS), is proposed to select stable feature variables for pattern recognition of vibrational spectroscopic data. First, some theoretical background for EBSS is introduced (Section 2). Then, to demonstrate the effectiveness of EBSS, the proposed algorithm was applied to four publicly available vibrational spectroscopic datasets (Section 3). The results of EBSS were compared with those obtained from single bootstrapping space shrinkage (BSS), GA-PLS-DA and sparse-PLS-DA (s-PLS-DA) (Section 4). Concluding remarks are given in Section 5.

\section{Theory}

\subsection{PLS-DA}

The theory and properties of the PLS-DA have been described elsewhere, ${ }^{33,34}$ hence only a short overview of the PLS-DA method, which is based on the PLS2 algorithm, is given here. Usually, the PLS-DA model is formulated as a regression equation:

$$
\mathbf{Y}=\mathbf{X B}
$$

where the independent variables are space $\mathbf{X}$ of size $N \times P$ and the regression coefficients are $\mathbf{B}$ of size $P \times J . N, P$ and $J$ stand for the numbers of samples, variables and classes, respectively. The Y matrix $(N \times J)$ of dependent variables contains information about class memberships of the objects; each row, $y^{\mathrm{T}}$, in the $\mathbf{Y}$ matrix has the following structure:

$$
y_{j}^{\mathrm{T}}\left\{\begin{array}{l}
1 \text { if object belongs to class } j \\
0 \text { otherwise }
\end{array}\right.
$$

where $y_{j}$ is the $j$ th column in $\mathbf{Y} . j$ is also the class number, where $j=1,2, \ldots, J$. The dummy $\mathbf{Y}$ matrix therefore has a structure where each row sums to unity.

Once the regression coefficients B are obtained, the prediction of dependent variables on a new set of objects can be done by

$$
\mathbf{Y}_{\text {test }}=\mathbf{X}_{\text {test }} \mathbf{B}
$$

However, the predicted values are real numbers and a conversion to class memberships is needed. In this study, the class membership of each unknown sample is assigned as the column index of the largest absolute value in the corresponding row of the $\mathbf{Y}_{\text {test }}$ matrix. ${ }^{5}$

\subsection{BSS}

The BSS is a basic predictor of EBSS, and it is also a modified version of BOSS. ${ }^{24}$ The BSS procedure can be summarized in the following steps:

For an independent variables space $\mathbf{X}$ with size $N \times P$ (contains $N$ samples, $P$ variables).

Step 1: the weighted bootstrap sampling (WBS) method $^{24}$ is applied to the whole variable space to generate $M($ e.g., 1000) variable subspaces. In each variables subspace, the repeated variables are excluded to remain unique. Note that the initial number of replacements in WBS is equal to $P$, and the initial sampling weight of each variable is set to $1 / P$. According to the bootstrap theory, the number of selected variables in each subset is about $0.632 P$.

Step 2: individual variable subspace is evaluated to determine its accuracy value using a PLS-DA algorithm and 10-fold cross validation is performed for extraction of the best variable subspaces $(10 \%)$ with the highest accuracy.

Step 3: the appearance frequency of each variable in the best variable subspaces is counted and the sampling weight of variable $p$ can then be updated as follows:

$$
w_{p}=\frac{f_{p}}{k_{\text {best }}}
$$

where $f_{p}$ represents the frequency of variable $p$ in the best variable subspaces, $k_{\text {best }}$ is the number of the best variable subspaces where $p=1,2, \ldots, P$. Let $w=\left[w_{1}, w_{2}, \ldots, w_{p}\right]$ and normalize the $w$.

Step 4: the number of replacements in WBS is updated and the value is determined by the average number of variables selected in the previous step. According to bootstrap theory, the number of variables in a new subset is about 0.632 times the previous one. Thus, variable space shrinks step by step.

Step 5: steps 1-4 are repeated until the average number of variables in the new subspaces equals the number of selected latent variables. The subspace with the best accuracy during the iteration is selected as the optimal variable set.

\subsection{EBSS}

The core idea of the EBSS algorithm is illustrated in Fig. 1. First, a set of subsets are generated from data set using random sampling. For an individual subset, a feature space is determined by BSS. Then an ensemble strategy and a sequential forward selection method are applied to the candidate feature spaces to select characteristic variables. The EBSS procedure can be summarized by the following steps:

Step 1: the data set is divided randomly into a training set $\mathbf{T}$ and a validation set $\mathbf{V}$. $\mathbf{T}$ consists of $67 \%$ of the data with $\mathbf{V}$ being the remainder. A feature space $\mathbf{F}$ is selected from $\mathbf{T}$ using the BSS method.

Step 2: repeat step $K$ times to give feature spaces $\mathbf{F}_{1}, \ldots, \mathbf{F}_{K}$.

Step 3: extract $R$ the most common recurring variables from the $K$ feature spaces based on eqn (4) (Section 2.2). 


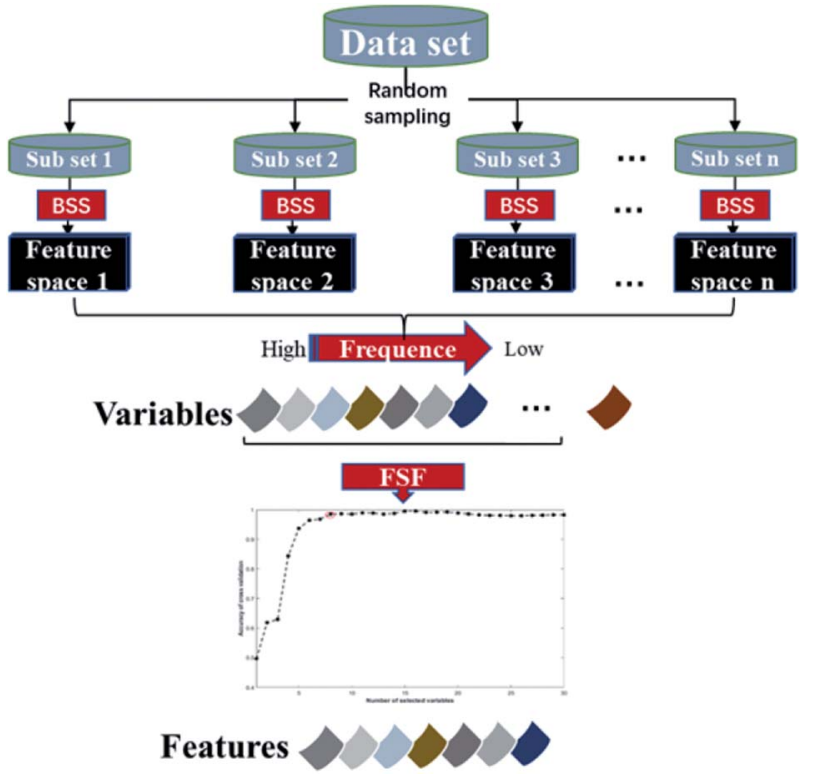

Fig. 1 The core idea of the EBSS algorithm.

Step 4: the final discriminant feature space $\mathbf{F}_{\mathbf{d}}$ is determined by running PLS-DA on training sets using from 1 to $\mathrm{R}$ of the most recurring variables and 10 -fold cross-validation.

Step 5: a PLS-DA is constructed from $\mathbf{T}_{i}$ using $\mathbf{F}_{\mathbf{d}}$. Running the validation set $\mathbf{V}_{i}$ down this PLS-DA gives an accuracy rate $a_{i}$
$\left(\mathbf{F}_{\mathbf{d}}, \mathbf{V}_{i}\right)$, where $i=1, \ldots, K$. The average accuracy rate $a_{\text {ave }}\left(\mathbf{F}_{\mathbf{d}}\right)$ and standard error $a_{\text {std }}\left(\mathbf{F}_{\mathbf{d}}\right)$ over the $K$ repeats are computed and recorded.

In this study, $K$ is set to $20, R$ is set to 30 (for Raman data, $R$ is set to 60$)$.

\subsection{Overview of the compared methods}

2.4.1 GA-PLS-DA. The GA-PLS-DA algorithm is a variable selection method of the PLS-DA based on the GA strategy. In the GA approach, a feature space is represented as a binary string (a chromosome) of length $P$, with a one or zero in position $p$ denoting the presence or absence of variable $p$. Note that $P$ is the total number of variables. A population of chromosomes is generated. Individual chromosome are evaluated to determine the fitness value, which determines how likely it is for the chromosome to survive and reproduce into next generation..$^{35}$ There are many different versions of the GA that perform reproduction, crossover, etc. in different ways. The algorithm applied in this study is based on the Genetic Algorithm Optimization Toolbox, ${ }^{36}$ which has several basic steps: (1) binary coding of the variables; (2) initiation of population; (3) performance evaluation of individuals; (4) selection of individuals; (5) recombination; (6) mutation; (7) reinsertion and (8) step 3 to step 7 are repeated until a maximum number of generations is reached. The parameters of the GA used in this study are listed in Table 1.

2.4.2 Sparse-PLS-DA. A s-PLS-DA method combines variable selection and classification in a one-step procedure. The s-

Table 1 Parameters for the GA-PLA-DA

Population size

Maximum number of generations

Generation gap

Crossover rate

Mutation rate

Maximum number of variables selected in the chromosome

Fitness value

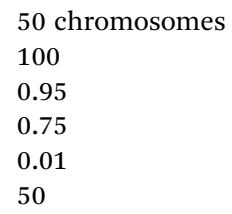

accuracy of 10-fold cross-validation of PLSDA

Table 2 Characteristics of the data sets

\begin{tabular}{llllr}
\hline Data set & Scan & $\begin{array}{l}\text { No. of training } \\
\text { samples }\end{array}$ & No. of test samples & No. of features \\
\hline Olive oils & FTIR & 82 & 38 & 570 \\
Red wines & FTIR & 30 & 14 & 842 \\
NIR tablets & NIR & 211 & 99 & 404 \\
Raman tablets & Raman & 82 & 38 & 3401
\end{tabular}

Table 3 Validation set accuracy $\left(a_{\text {ave }} \pm a_{\text {std }} \%\right)^{a}$

\begin{tabular}{llllll}
\hline Data set & Type & PLS-DA & BSS & GA-PLS-DA & s-PLS-DA \\
\hline Olive oil & FTIR & $93.2 \pm 2.2$ & $94.7 \pm 2.6$ & $93.6 \pm 3.1$ & $95.1 \pm 3.1$ \\
Red wine & FTIR & $59.3 \pm 14.3$ & $60 \pm 13.4$ & $60.4 \pm 9.4$ & $66.8 \pm 9.6$ \\
NIR tablet & NIR & $88.9 \pm 2.5$ & $87 \pm 3.6$ & $86.4 \pm 3.4$ & $88.6 \pm 3.2$ \\
Raman tablet & Raman & $85.8 \pm 5.7$ & $81.4 \pm 4.2$ & $80.4 \pm 4.7$ & $78.8 \pm 4.9$
\end{tabular}

${ }^{a} a_{\text {ave }} \pm a_{\text {std }}:$ average accuracy rate \pm standard error over 20 repeats. 
Table 4 The number of selected variables $\left(n_{\text {ave }} \pm n_{\text {std }}\right)^{a}$

\begin{tabular}{llrlllr}
\hline Data set & Type & PLS-DA & BSS & GA-PLS-DA & s-PLS-DA & EBSS \\
\hline Olive oil & FTIR & 570 & $34 \pm 33$ & $29 \pm 10$ & $69 \pm 22$ & 8 \\
Red wine & FTIR & 842 & $43 \pm 34$ & $33 \pm 15$ & $52 \pm 31$ & 21 \\
NIR tablet & NIR & 404 & $46 \pm 21$ & $44 \pm 8$ & $59 \pm 18$ & 20 \\
Raman tablet & Raman & 3041 & $58 \pm 22$ & $60 \pm 8$ & $77 \pm 19$ & 40
\end{tabular}

${ }^{a} n_{\text {ave }} \pm n_{\text {std: }}$ average number of selected variable \pm standard error over 20 repeats.

PLS-DA algorithm used in this study was proposed by Ewa Szymanska et al. ${ }^{18}$ details of which are provided elsewhere. ${ }^{\mathbf{1 1}, 18}$ There are two parameters to be considered in the s-PLSDA: the number of latent variables and the number of selected variables for each latent variable. In this study, the maximum number of latent variables was set to 10 and the number of selected variables for each latent variable was also set to 10 . The variables with the best prediction ability were recorded.

\subsection{Algorithm evaluation}

The EBSS algorithm was evaluated by the procedure as described in Section 2.3. For BSS, GA-PLS-DA and S-PLS-DA, each algorithm was evaluated, independently, in the following way:
Step 1: the data set was randomly divided into a training set $\mathbf{T}$ and a validation set $\mathbf{V}$. T consisted of $67 \%$ of the data, and $\mathbf{V}$ the remainder.

Step 2: a feature space $\mathbf{F}$ was selected from $\mathbf{T}$ using the variable selection method. A PLS-DA model was constructed from $\mathbf{T}$ using $\mathbf{F}$. Running the validation set $\mathbf{V}$ down this PLS-DA gave the accuracy rate $a(\mathbf{F}, \mathbf{V})$.

Step 3: steps 1 and 2 were repeated 20 times giving feature spaces $\mathbf{F}_{\mathbf{1}}, \ldots, \mathbf{F}_{20}$ and accuracy rates $a_{1}\left(\mathbf{F}_{\mathbf{1}}, \mathbf{V}_{\mathbf{1}}\right), \ldots, a_{20}\left(\mathbf{F}_{20}, \mathbf{V}_{20}\right)$. The average accuracy rate $a_{\text {ave }}$ and the standard error $a_{\text {std }}$ over the 20 repeat were computed and recorded.

For all of the algorithms the optimal number of latent variables for PLS-DA model was determined by 10 -fold cross-validation, the data set was mean-centered before modeling. ${ }^{16}$

\section{Data sets and experimental condition}

\subsection{Data sets}

3.1.1 IR data of olive oils. The olive oil data set was downloaded from http://asu.ifr.ac.uk/example-datasets-fordownload/. The website contains digitized IR spectra for 120 authenticated extra virgin olive oils samples which originated from four producing countries corresponding to four different classes of olive oil. ${ }^{1}$ The spectra in this dataset were recorded within the range $799-1897 \mathrm{~cm}^{-1}$. (a)

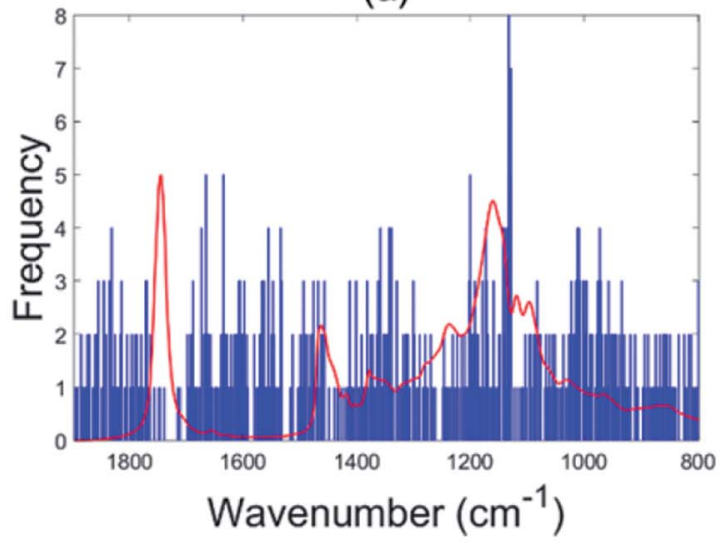

(c)

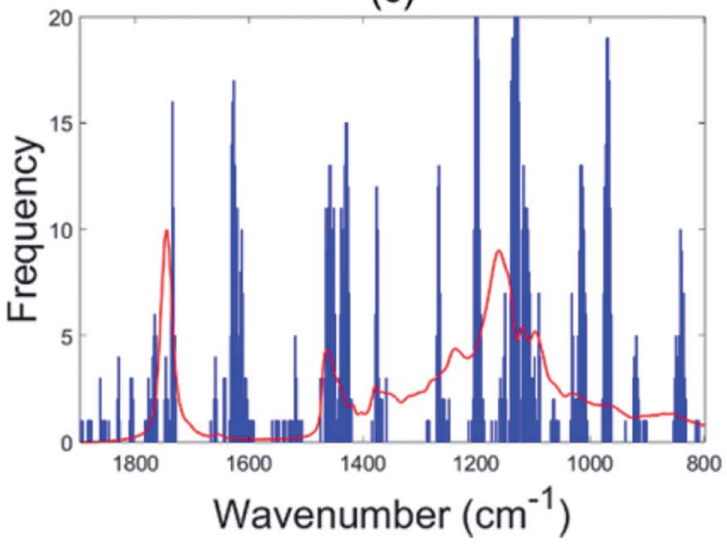

(b)

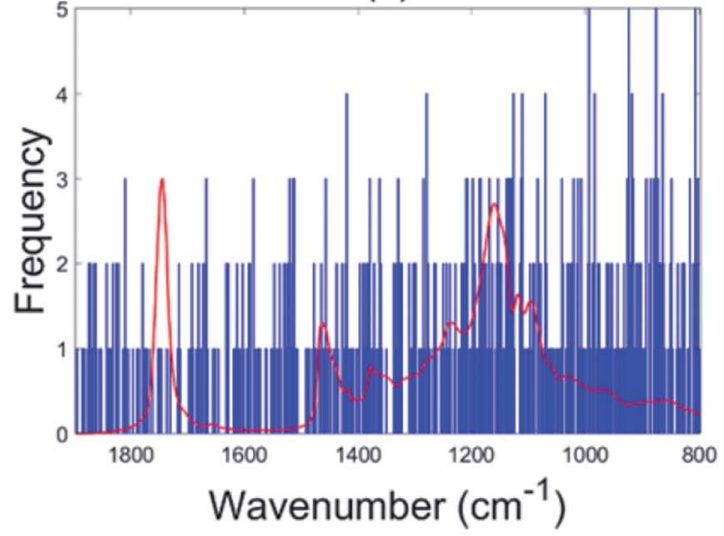

(d)

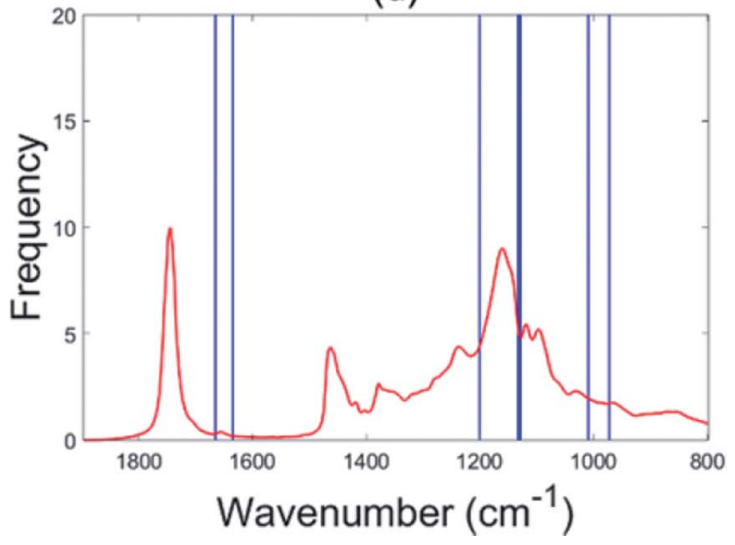

Fig. 2 Variables selected by the different methods for the olive oil data: BSS (a), GA-PLS-DA (b), s-PLS-DA (c) and EBSS (d). 


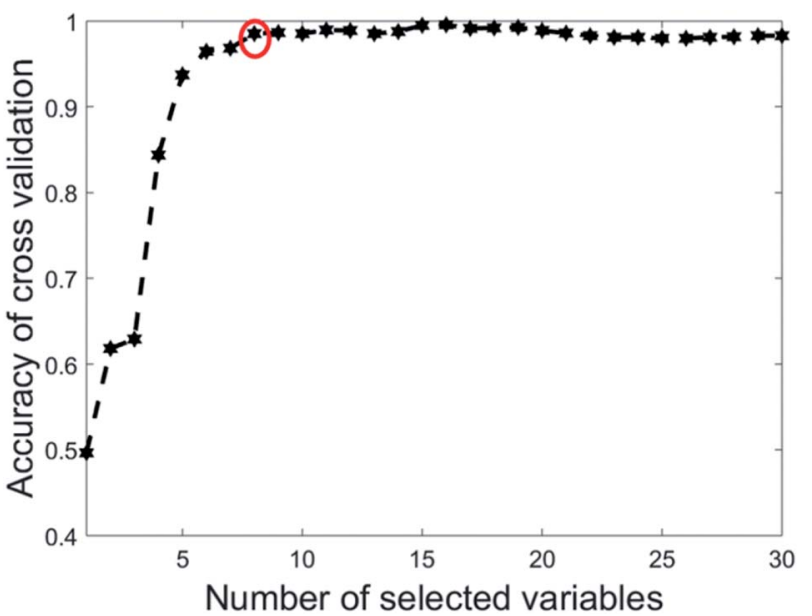

Fig. 3 Effect of number of variables selected by EBSS on the accuracy for the olive oil data.

3.1.2 IR data of red wines. This data set was downloaded from http://www.models.life.ku.dk/Wine_GCMS_FTIR. The data represent the FT-IR spectra for 44 red wine samples prepared exclusively from $100 \%$ the Cabernet Sauvignon grapes and harvested in four different geographical regions, ${ }^{37}$ i.e., the four classes of wine originated from four different regions.
3.1.3 NIR and Raman data of pharmaceutical tablets. NIR and Raman spectra were downloaded from: http:// www.models.life.ku.dk/Tablets. The samples were grouped into four different categories of tablets, each category containing different amounts of active substance. ${ }^{38}$ The NIR spectra of the tablets were recorded in the range 4000-14 $000 \mathrm{~cm}^{-1}$, Altogether there were 310 samples. Raman spectra were collected in the range 200-3600 $\mathrm{cm}^{-1}$. Altogether there were 120 samples.

An overview of the characteristics of the different data sets is given in Table 2. For each data set, $67 \%$ of samples were randomly selected for the training set and those remaining were used as a validation set. ${ }^{24}$

\subsection{Experimental conditions}

All computations were performed in MATLAB (Version 2016a, MathWorks, Inc.) on a personal computer (Intel Core i7-7700 $3.6 \mathrm{GHz}$ CPU and $8 \mathrm{~GB}$ RAM). MATLAB codes for s-PLS-DA were acquired courtesy of Ewa Szymanska. The GA-PLS-DA, BSS and EBSS algorithms were realized with home-made codes which are available upon request.

\section{Results and discussion}

Table 3 gives the validation set accuracies for the different methods. The data in bold denote the best performance on each data set. (a)

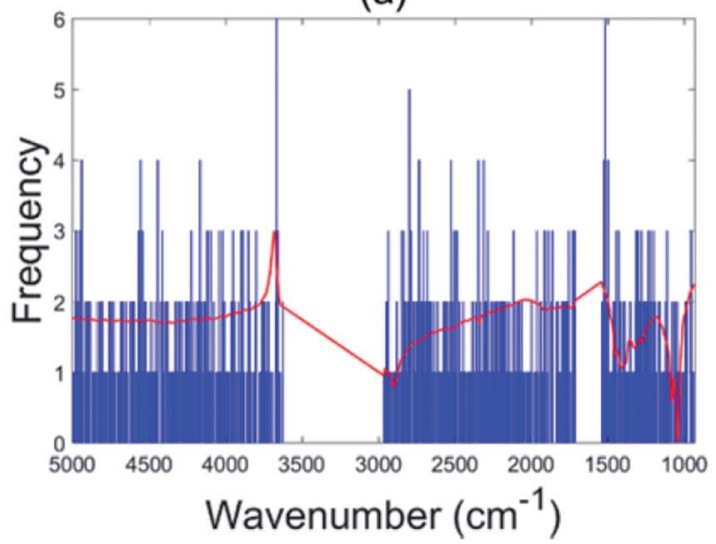

(c)

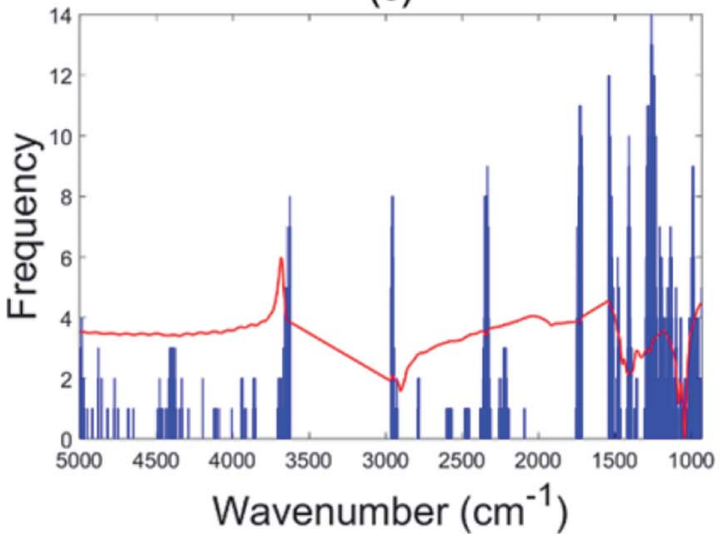

(b)

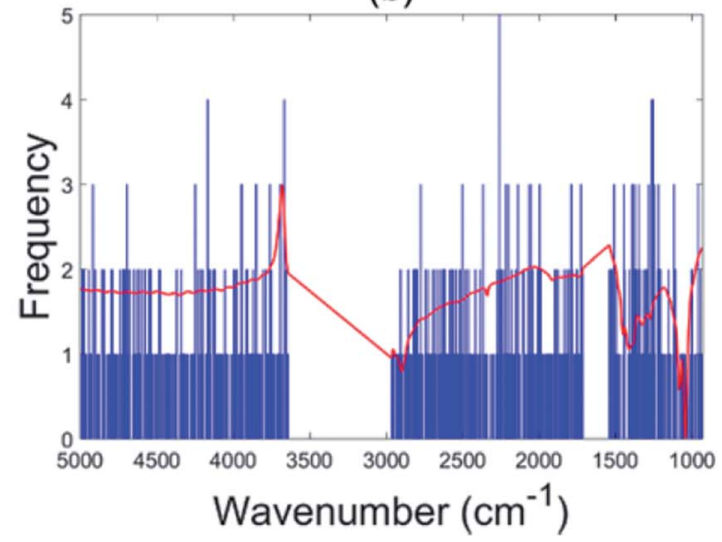

(d)

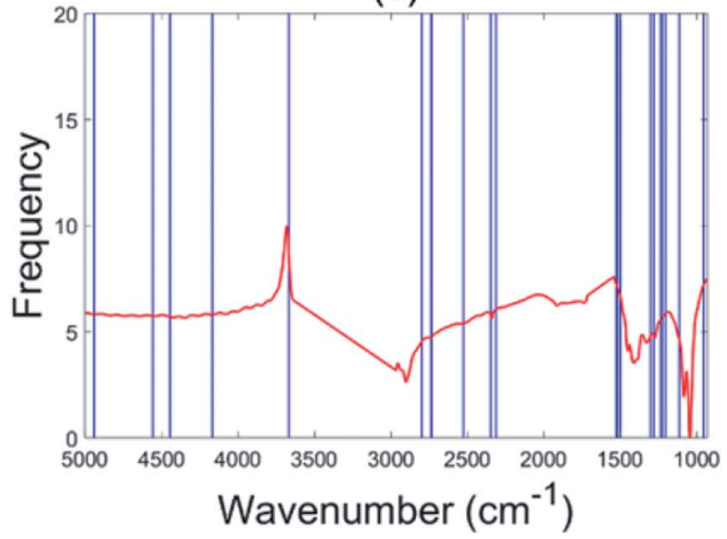

Fig. 4 Variables selected by the different methods for the red wine data set: BSS (a), GA-PLS-DA (b), s-PLS-DA (c) and EBSS (d). 


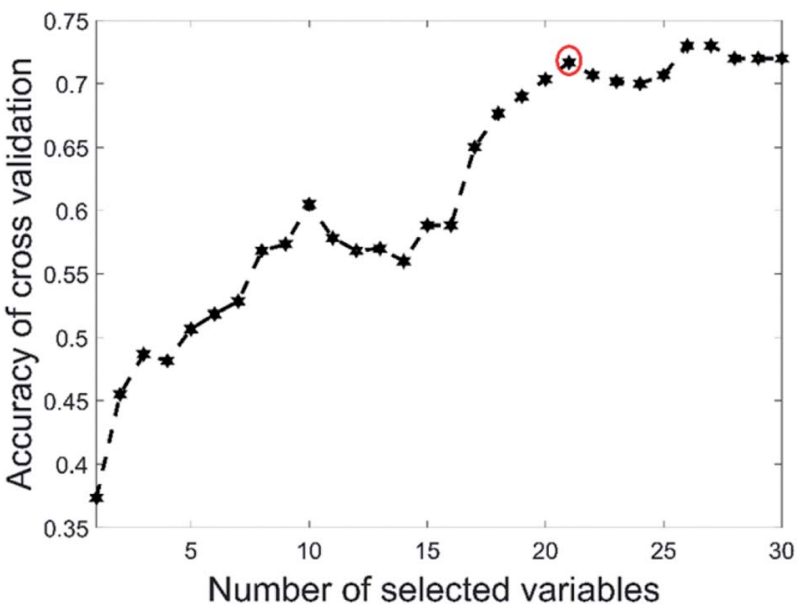

Fig. 5 Effect of number of variables selected by EBSS on the accuracy for the red wine data.

As Table 3 shows, EBSS gives the best performance relative to the other methods for all datasets. The number of selected variables for each method is given in Table 4 . It can be seen that the EBSS method used fewer variables than the other methods.

\subsection{Olive oil data set}

For the olive oil data set, the accuracy was improved from $93.2 \%$ in the case of PLS-DA to $96.6 \%$ for the EBSS method. Other variable selection methods also show better classification performance than the full range PLS-DA (BSS: 94.7\%, GA-PLSDA: $93.6 \%$, s-PLS-DA: $95.1 \%$ ), which demonstrates the benefit of conducting variable selection.

The selected wavenumber variables are displayed in Fig. 2. In each subgraph the $x$-axis represents the wavenumber variables and the $y$-axis represents the frequency of each variable selected by the algorithm after 20 repeat operations. Instability of the selected sets of informative variables can be found when using BSS and GA-PLA-DA given that these selection methods are guided by a random search. The stability of variable selection performed with s-PLS-DA was better than those for BSS and GAPLS-DA. For EBSS, eight variables were selected to discriminate between four classes. The eight wavenumbers were 966.8, 1003.4, 1123.1, 1125.0, 1126.9, 1194.1, 1628.6 and $1665.3 \mathrm{~cm}^{-1}$ (see Fig. 2d).

The eight wavenumbers were determined by running PLS-DA using 1 to 30 as the most recurring variables (see Section 2.3). In each iteration, $67 \%$ of the data was split off as a training set. Then $n$ VAR, the number of variables selected was varied from 1 to 30 . For each value of $n \mathrm{VAR}$, a PLS-DA model was constructed (a)

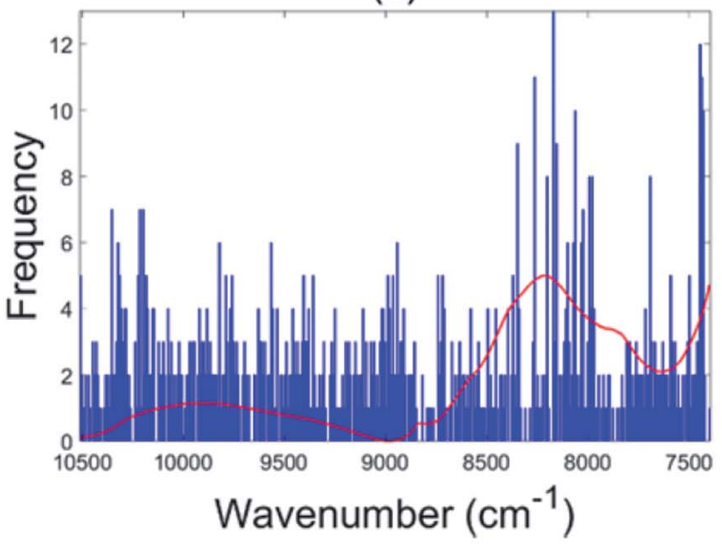

(c)

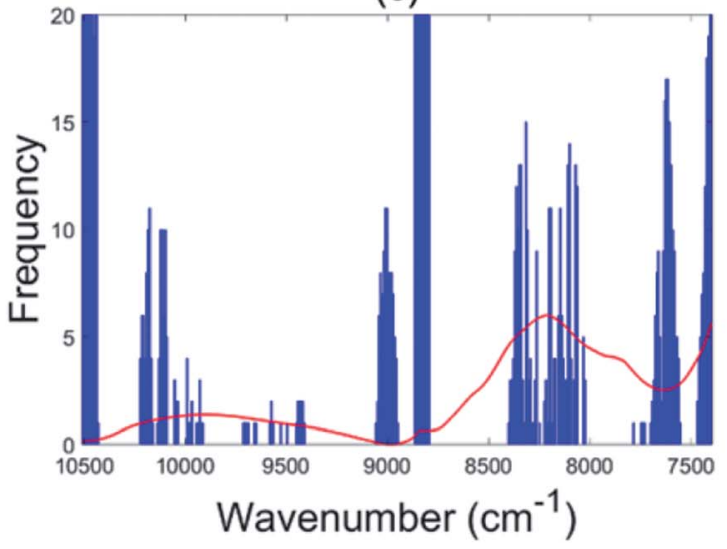

(b)

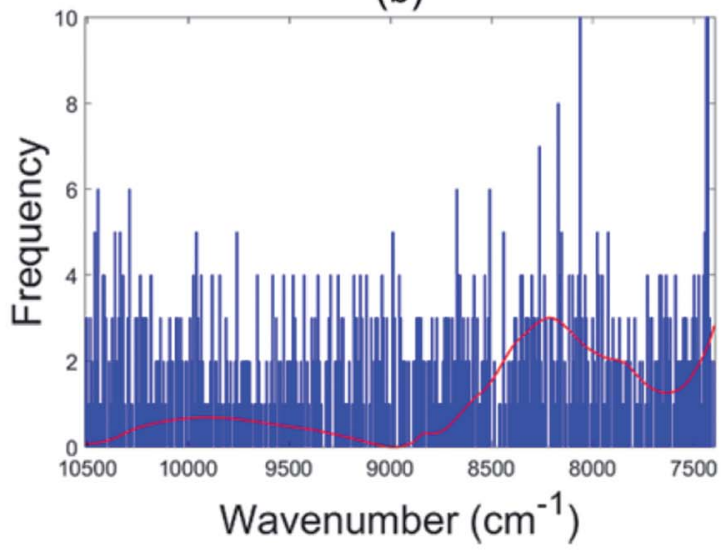

(d)

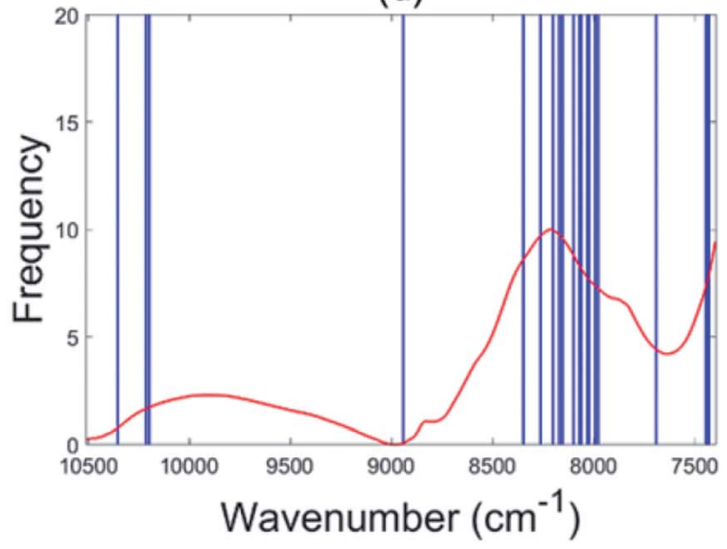

Fig. 6 Variables selected by the different methods for the NIR tablet data: BSS (a), GA-PLS-DA (b), s-PLS-DA (c) and EBSS (d). 
(a)

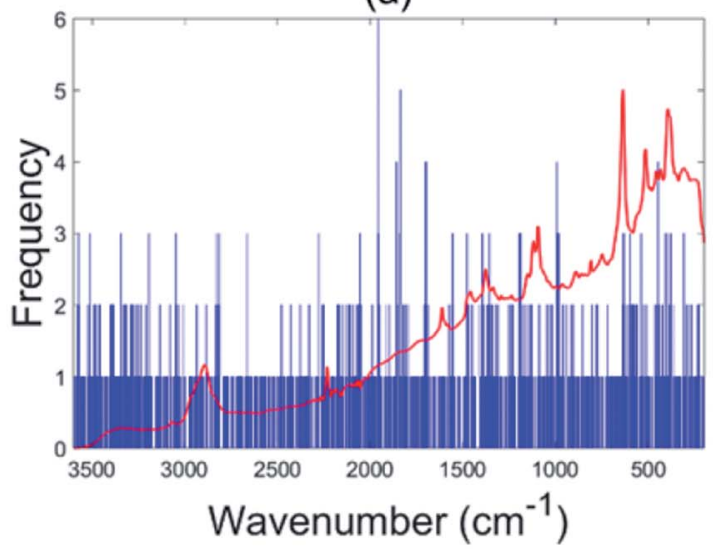

(c)

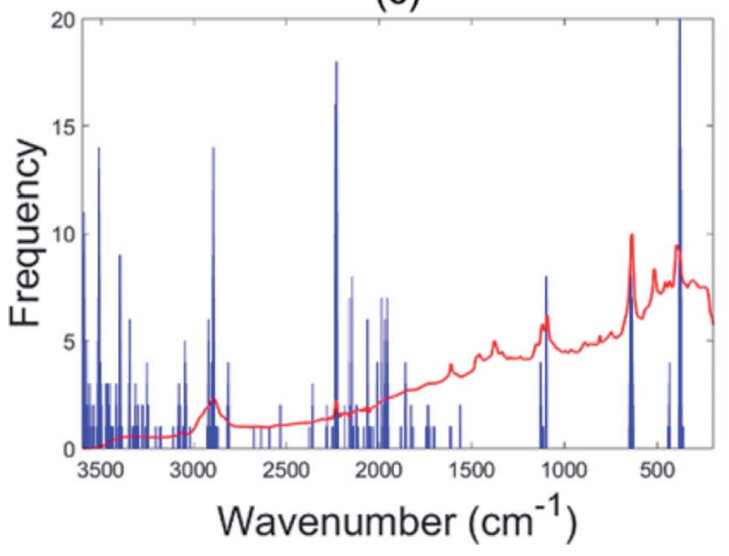

(b)

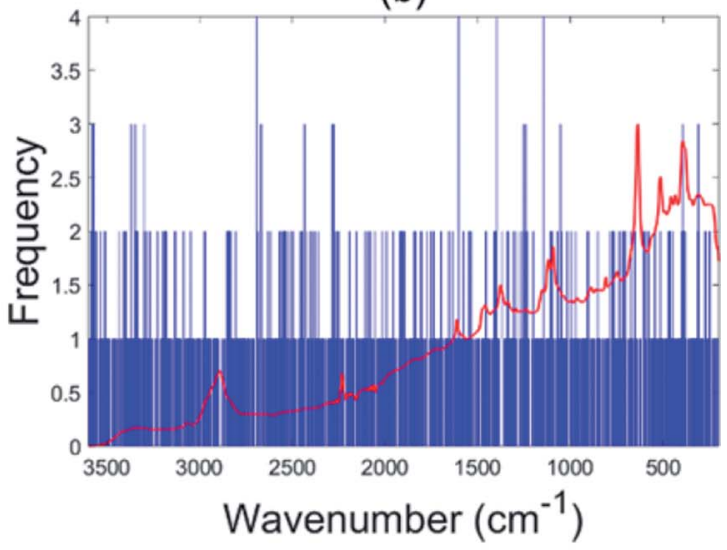

(d)

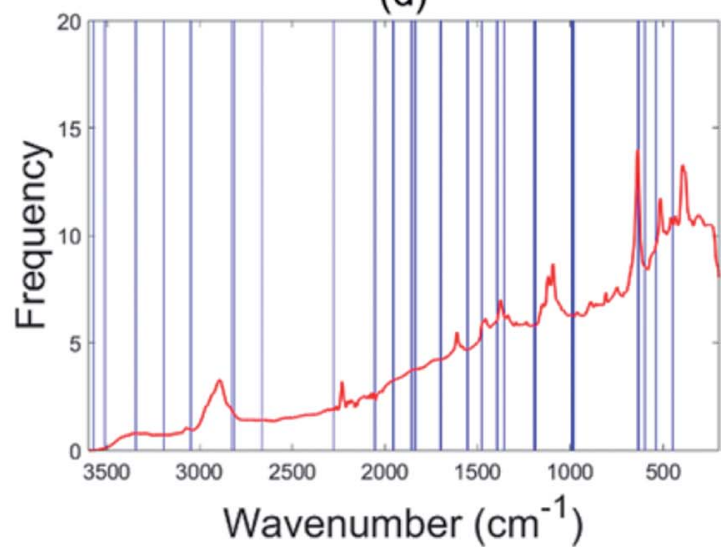

Fig. 7 Variables selected by the different methods for the Raman tablet data: BSS (a), GA-PLS-DA (b), s-PLS-DA (c) and EBSS (d).

on the training set using 10 -fold cross-validation and then the accuracy of cross-validation was recorded. Twenty iterations were done, and the accuracy values were averaged over 20 repetitions. Fig. 3 is a plot of the accuracy of cross-validation $v s$. $n$ VAR. The result is fascinating. The cross-validation accuracy increases from $n \mathrm{VAR}=1$ to $n \mathrm{VAR}=8$. Beyond about $n \mathrm{VAR}=8$ the accuracy remained constant, i.e., adding more variables did not improve accuracy.

\subsection{Red wine data set}

This data set had only 44 as the total number of samples with BSS giving a validation set accuracy of $60 \%$ and GA-PLS-DA had an accuracy of $60.4 \%$. The s-PLS-DA had an accuracy of $66.8 \%$, which was a $12.6 \%$ improvement compared with $59.3 \%$ using full range PLS-DA. The EBSS method gave an accuracy of $71.1 \%$, an improvement of $19.9 \%$. These results illustrate that variable selection is necessary to improve the separation ability, and that PLS-DA may not be a good choice for small samples datasets. ${ }^{39}$

Fig. 4 shows the selected variables for the different methods. The BSS, GA-PLS-DA and s-PLS-DA methods produced different feature spaces in each repeat. In total, 21 wavenumber variables were selected by EBSS. The selected variables are also listed in the Appendix (Table 5). The way that was used to reserve the 21 variables was the same as that for the olive oil dataset. Fig. 5 is a plot of the accuracy of crossvalidation $v s$. $n$ VAR. The accuracy values increased from $n \mathrm{VAR}=1$ to $n \mathrm{VAR}=10$, a small drop occurred from $n \mathrm{VAR}=11$ to $n \mathrm{VAR}=14$, then the accuracy gradually increased and remained constant beyond about $n \mathrm{VAR}=21$.

\subsection{Tablet data sets}

The EBSS method gave better performance than full range PLSDA for both the NIR and Raman tablet data sets. For the NIR tablet data set, the EBSS model had an $89.3 \%$ validation accuracy based on only 20 selected variables (Fig. 6d). For the Raman tablet data set, the EBSS model was found to have an $89.3 \%$ validation accuracy and the total number of selected variables was 40 (Fig. 7d). From Fig. 6 and 7, it can also be observed that the wavelengths selected by BSS, GA-PLS-DA and s-PLS-DA for the NIR and Raman data sets were labile.

Fig. 8 shows the effect of the number of selected variables on the accuracy of cross-validation. For the NIR tablet data, the accuracies steadily increased from $n \mathrm{VAR}=1$ to $n \operatorname{VAR}($ e.g., about 20 ), and then remained constant (see Fig. 8a). For the Raman tablet data, the number of selected variables was varied from 1 to 60 . Again, the accuracies showed a steady increase from $n$ VAR $=1$ to $n \mathrm{VAR}=40$, and then remained constant (see Fig. 8b). 


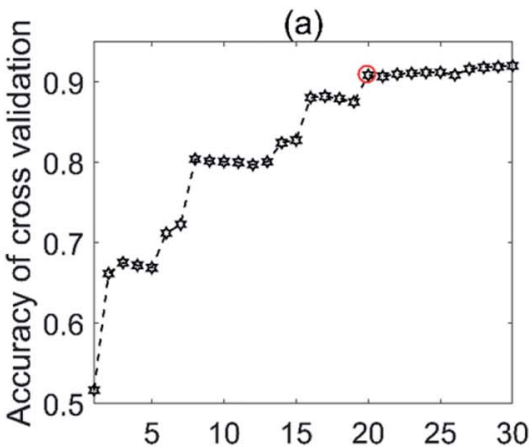

Number of selected variables

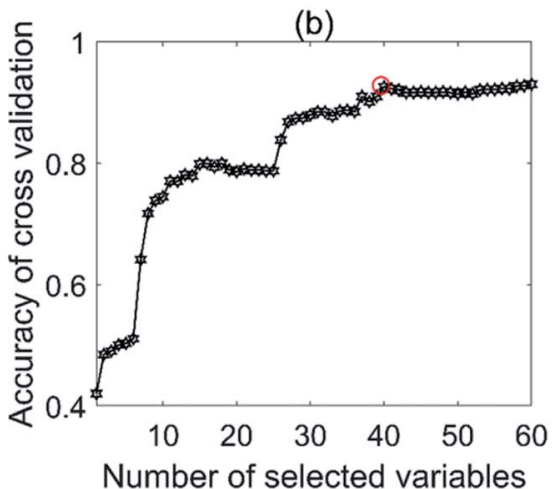

Fig. 8 Effect of selected variables on the accuracy for the tablet data sets: (a) NIR and (b) Raman.

As the above results demonstrated, the EBSS method was superior to GA-PLS-DA, BSS and s-PLS-DA in terms of performance.

\section{Conclusion}

A method termed EBSS, which feature contains bootstrap sampling and an ensemble strategy, has been proposed to select characteristic variables for pattern recognition of vibrational spectroscopic data. The results suggest that the new EBSS algorithm can reserve more stable and informative variables for the final modeling and provide better performance than those obtained from GA-PLS-DA, BSS and s-PLS-DA.

The investigations also suggest that EBSS can be an alternative method for rapid classification problem solving with IR, NIR and Raman spectroscopic data. There are many important potential application areas for the EBSS method, such as in biotechnology, food science and medicine, where there is an increasing interest in using atomic and molecular spectroscopies for rapid screening purposes. A challenge is to identify a stable and small number of wavelengths and incorporate into low-cost and accurate instruments tailored to solving specific screening problems.

\section{Appendix}

Table 5 Selected variables for the four different data sets using EBSS

\begin{tabular}{ll}
\hline Data set & Wavenumber $\left(\mathrm{cm}^{-1}\right)$ \\
\hline Olive oil & $966.8,1003.4,1123.1,1125.0,1126.9,1194.1,1628.6$, \\
& 1665.3 \\
Red wine & $956.0,1114.1,1202.8,1222.0,1237.5,1279.9,1303.0$, \\
& $1499.6,1518.9,1526.6,2313.0,2347.7,2525.0,2733.2$, \\
& $2737.1,2798.7,3666.1,4167.3,4444.8,4556.6,4919.0$ \\
Tablet & $7429.2,7436.9,7444.6,7691.5,7976.9,7992.4,8023.2$, \\
(NIR) & $8030.9,8061.8,8069.5,8100.4,8154.4,8169.8,8200.6$, \\
& $8347.2,8941.2,10198.7,10214.1,10353.0$ \\
Tablet & $3575,3514,3345,3192,3048,3047,2826,2816,2666$, \\
(Raman) & $2279,2058,2056,1957,1955,1954,1858,1840,1839$, \\
& $1838,1703,1701,1699,1556,1477,1356,1395,1196$, \\
& $1194,1193,1191,1190,993,989,983,982,639,632,597$, \\
& 540,449
\end{tabular}

\section{Conflicts of interest}

There are no conflicts to declare.

\section{Acknowledgements}

The authors would like to thank Ewa Szymanska, from Radboud University Nijmegen, The Netherlands for providing the sparsePLS-DA codes. We also grateful to Kuangda Tian for providing helpful suggestions for our research.

\section{References}

1 H. S. Tapp, A. Marianne Defernez and E. K. Kemsley, J. Agric. Food Chem., 2003, 51, 6110-6115.

2 R. M. Balabin, R. Z. Safieva and E. I. Lomakina, Anal. Chim. Acta, 2010, 671, 27-35.

3 Y. Roggo, K. Degardin and P. Margot, Talanta, 2010, 81, 988995.

4 K. Degardin, Y. Roggo, F. Been and P. Margot, Anal. Chim. Acta, 2011, 705, 334-341.

5 B. K. Alsberg, D. B. Kell, R. Goodacre and A. Chem, Anal. Chem., 1998, 70, 4126-4133.

6 Z. Ramadan, D. Jacobs, M. Grigorov and S. Kochhar, Talanta, 2006, 68, 1683-1691.

7 R. G. Brereton, Chemom. Intell. Lab. Syst., 2015, 149, 90-96. 8 T. Mehmood, K. H. Liland, L. Snipen and S. Sæbø, Chemom. Intell. Lab. Syst., 2012, 118, 62-69.

9 A. Höskuldsson, Chemom. Intell. Lab. Syst., 2001, 55, 23-38. 10 A. L. Boulesteix and K. Strimmer, Briefings Bioinf., 2007, 8, 32.

11 K. A. L. Cao, S. Boitard and P. Besse, BMC Bioinf., 2011, 12, 253.

12 T. Mehmood, H. Martens, S. Sæbø, J. Warringer and L. Snipen, Algorithms Mol. Biol., 2011, 6, 27.

13 G. Quintás, N. Portillo, J. C. García-Cañaveras, J. V. Castell, A. Ferrer and A. Lahoz, Metabolomics, 2011, 8, 86-98.

14 X.-M. Sun, X.-P. Yu, Y. Liu, L. Xu and D.-L. Di, Chemom. Intell. Lab. Syst., 2012, 115, 37-43.

15 J. Kuligowski, D. Perez-Guaita, J. Escobar, M. de la Guardia, M. Vento, A. Ferrer and G. Quintas, Talanta, 2013, 116, 835840. 
16 Y.-Q. Li, Y.-F. Liu, D.-D. Song, Y.-P. Zhou, L. Wang, S. Xu and Y.-F. Cui, Chemom. Intell. Lab. Syst., 2014, 135, 192-200.

17 Y.-F. Liu, S. Xu, H. Gong, Y.-F. Cui, D.-D. Song and Y.-P. Zhou, J. Chemom., 2015, 29, 537-546.

18 E. Szymanska, E. Brodrick, M. Williams, A. N. Davies, H. J. van Manen and L. M. Buydens, Anal. Chem., 2015, 87, 869-875.

19 H. Xie, J. Zhao, Q. Wang, Y. Sui, J. Wang, X. Yang, X. Zhang and C. Liang, Sci. Rep., 2015, 5, 10930.

20 G. Aliakbarzadeh, H. Parastar and H. Sereshti, Chemom. Intell. Lab. Syst., 2016, 158, 165-173.

21 L. Deng, H. Gu, J. Zhu, G. A. Nagana Gowda, D. Djukovic, E. G. Chiorean and D. Raftery, Anal. Chem., 2016, 88, 79757983.

22 S. Kasemsumran, N. Suttiwijitpukdee and V. Keeratinijakal, Anal. Sci., 2017, 33, 111-115.

23 Y. Saeys, I. Inza and P. Larranaga, Bioinformatics, 2007, 23, 2507-2517.

24 B. C. Deng, Y. H. Yun, D. S. Cao, Y. L. Yin, W. T. Wang, H. M. Lu, Q. Y. Luo and Y. Z. Liang, Anal. Chim. Acta, 2016, 908, 63-74.

25 X. Song, Y. Huang, H. Yan, Y. Xiong and S. Min, Anal. Chim. Acta, 2016, 948, 19-29.

26 W. Zheng, X. Fu and Y. Ying, J. Chemom., 2017, 31, e2893.
27 N. Meinshausen and P. Bühlmann, J. Royal Stat. Soc., 2010, 72, 417-473.

28 L. Breiman, Mach. Learn., 1996, 24, 123-140.

29 L. Breiman, Mach. Learn., 2001, 45, 5-32.

30 T. G. Dietterich, Proc International Workshop on Multiple Classifier Systems, 2000, vol. 1857, pp. 1-15.

31 T. G. Dietterich, Mach. Learn., 2000, 40, 139-157.

32 Q. J. Han, H. L. Wu, C. B. Cai, L. Xu and R. Q. Yu, Anal. Chim. Acta, 2008, 612, 121-125.

33 M. Barker and W. Rayens, J. Chemom., 2003, 17, 166-173.

34 R. G. Brereton and G. R. Lloyd, J. Chemom., 2014, 28, 213225.

35 H. c. C. Goicoechea and A. C. Olivieri, J. Chemom., 2003, 17, 338-345.

36 A. J. Chipperfield and P. J. Fleming, IEEE Colloquium on Applied Control Techniques Using MATLAB, 1995.

37 T. Skov, D. Ballabio and R. Bro, Anal. Chim. Acta, 2008, 615, 18-29.

38 M. Dyrby, S. B. Engelsen, L. Nørgaard, M. Bruhn and L. Lundsbergnielsen, Appl. Spectrosc., 2002, 56, 579-585.

39 J. Acquarelli, T. van Laarhoven, J. Gerretzen, T. N. Tran, L. M. C. Buydens and E. Marchiori, Anal. Chim. Acta, 2017, 954, 22-31. 